Voix et Images

voixetimages

\title{
Fernand Leduc : de l'automatisme aux microchromies
}

\section{Jean-Pierre Duquette}

Volume 2, numéro 1, septembre 1976

Fernand Leduc

URI : https://id.erudit.org/iderudit/200016ar

DOI : https://doi.org/10.7202/200016ar

Aller au sommaire du numéro

Éditeur(s)

Les Presses de l'Université du Québec

ISSN

0318-9201 (imprimé)

1705-933X (numérique)

Découvrir la revue

Citer ce document

Duquette, J.-P. (1976). Fernand Leduc : de l'automatisme aux microchromies.

Voix et Images, 2(1), 3-12. https://doi.org/10.7202/200016ar d'utilisation que vous pouvez consulter en ligne.

https://apropos.erudit.org/fr/usagers/politique-dutilisation/ 


\section{Fernand Leduc: de l'automatisme aux microchromies}

On a beaucoup parlé, ces récentes années, de la révolution qu'apportaient les idées et les couvres du groupe automatiste a Montréal dans les années d'après-guerre 1. Deux expositions capitales: celle de la rue Amherst en avril 1946; et celle du 75 ouest, rue Sherbrooke, en février 1947, qui réunissait des tableaux de Borduas, Barbeau, Fauteux, Gauvreau, Leduc et Mousseau. C'est du reste dans un compte rendu de cette exposition que Tancredde Marsil parla, le premier, de "groupe automatiste " (Quartier latin, 28 fevvrier 1947), à partir du titre d'un tableau de Borduas: Automatisme 1.47.

Dans sa propriété de la campagne de Chartres, Fernand Leduc a répondu à mes questions sur son rôle au sein du groupe automatiste et sur l'évolution de son œuvre personnelle jusqu'aujourd'hui. Et au cours de notre conversation, sa femme, Thérèse Renaud, a bien voulu se joindre a nous pour rappeler l'histoire de son fameux recueil de poèmes, les Sables du rêve. (J.-P. Duquette)

F. L. Le mot automatisme a surgi dans une critique, il a "collé " à la réalité des tableaux et des idées esthétiques de notre groupe, et on l'a gardé. Quant à savoir s'il a été inspiré à Marsil par le titre d'une toile de Borduas, j'ai des doutes: je n'ai aucun souvenir d'un tel titre à l'époque... C'est là un de ces phénomènes qui surgissent à un moment donné, et dans lesquels on essaie de voir clair après, auxquels on cherche - et on donne - des explications plausibles...

J.-P. D. Vous avez fait la connaissance de Borduas à l'automne de 1941; en mai 1943 avait lieu l'exposition des Sagittaires, à la Galerie Dominion: vingt-trois jeunes artistes. Quel est le pôle d'attraction qui réunissait alors ces jeunes? Peut-on voir dans le groupe - un peu fortuit - des Sagittaires le noyau ou l'embryon de ce qui sera bientôt après les Automatistes?

F. L (me montrant une photo parue dans la Presse du 1er mai 1943): Voici, la veille du vernissage, quelques-uns des exposants autour de deux personnages importants de l'époque: le Père Couturier et Maurice Gagnon, qui avaient organisé cette exposition après en avoir eu l'idée ensemble, avec Borduas. II s'agissait alors d'une préoccupation essentielle: mettre sur pied une manifestation d' "art vivant", contre tous les académismes et les expressions figées, immobiles. On réunirait de jeunes artistes de disciplines diverses, encore inconnus pour la plupart, et qui constituaient 
aux yeux des organisateurs la relève, une succession vivante et dynamique dans la production artistique de l'époque. L'exposition ne comportait aucun tableau de Borduas, mais il était présent partout: la majeure partie des exposants était formée de ses étudiants à l'École du meuble (Maisonneuve, Guy Viau, Gilles Charbonneau, F. Vinet, L. Labrèche, Charles Daudelin, Marcel Coutlée, André Jasmin, entre autres); ou encore de ses disciples, et de quelques élèves et anciens élèves de l'École des beaux-arts (Yvonne Roy et Jeanne Rhéaume, qui avaient déjà un certain renom; A. Villandré, Louise Renaud, Françoise Sullivan, Pierre Gauvreau, Madeleine Desroches, Lucien Morin, Fernand Leduc). Donc, une exposition qui voulait témoigner d'un «art vivant», encore une fois, par opposition à l'art académique. En fait, ces œuvres diverses n'avaient pas d'inspiration commune; on y voyait aussi bien du figuratisme naïf, des portraits ou des paysages. Mais à part quelques exceptions, tous étaient des inconnus. On ne peut voir chez les Sagittaires d'annonce du groupe automatiste, sinon dans leur prise de position contre l'enseignement des beaux-arts et toute institution académique, traditionnelle. La Galerie Dominion prenait évidemment un risque important - et conscient - en organisant cette exposition qui ne suscita à vrai dire pas de grands remous: quelques journalistes en parlèrent, et aussi, bien entendu, Maurice Gagnon et Charles Doyon. En tout cas, un bouillonnement était dans l'air, dès ce printemps de 1943.

J.-P.D. Peu après cette exposition, au cours de l'hiver 1943-1944, vous donnez au Quartier latin six articles "retentissants" qui constituent une sorte de base théorique des idées qui réuniront les Automatistes. On parlera de Leduc comme du "théoricien" et du "propagandiste" du groupe, début 1946. Accepte-t-il ce titre aujourd'hui?

F. L. Cette série d'articles constitue peut-être une première rédaction cohérente menant aux théories automatistes. Mais il y avait déjà des textes importants. En particulier la conférence de Borduas intitulée «Des mille manières de goûter une cuvre d'art". Cinq d'entre nous l'avaient entendue à l'hiver de 1942 (Gauvreau, Madeleine Desroches, Louise Renaud, Françoise Sullivan et moi). Le texte parut dans Amérique française en janvier 1943 (vol. 2, n 4). C'était là l'exposé des idées essentielles de Borduas sur l'œuvre d'art. II y eut également des critiques de Robert Élie, de Pariseau. Donc, déjà, quelques textes qui allaient alimenter notre réflexion.

Quant à mes articles du Quartier latin, peut-on parler ici à mon sujet de "théoricien et propagandiste» de ce qui sera l'automatisme? II faut préciser d'abord que de par ma formation (École normale, École des beaux-arts) et mon activité (enseignement), j'avais sans doute un tempérament plus didactique que les autres. C'est ainsi que j'ai été amené à vouloir fixer par écrit les éléments principaux de nos discussions. J'éprouvais toujours le besoin de faire le point, d'exposer clairement les idées qui se dégageaient de nos rencontres. Cela dit, je ne me considère absolument pas comme le porte-drapeau des Automatistes; mais, 
dans les circonstances, je voulais noter et ordonner le fruit de nos échanges. Dans mon premier article par exemple, je posais la question: l'artiste est-il un être anormal?, dans le sens où on l'entendait alors au Québec (les artistes sont des fous, des hurluberlus, etc.). Je tentais de définir le rôle de l'artiste dans la société en précisant que toute création authentique est "différente», donc bousculante: l'artiste véritable est au cœur de la vie même. Est-il seulement vraisemblable qu'on le qualifie d'anormal? J'écrivais: "L'artiste n'est en marge de la société que lorsque celle-ci est en marge de la vie. " Je le pense toujours.

J.-P. D. Commentant cette série d'articles, Bernard Teyssèdre parle de «virulence extrémiste " en même temps que de “pondération d'un caractère qui aspire intensément à l'ordre "...

F. L Oui, je pense que c'est juste. Cette sorte de balancement, de besoin d'équilibre, je les tiens de mon éducation, de ma formation. Cela fait partie de mon caractère même. Je serai l'un des premiers, par exemple, à m'éloigner de «l'accident" automatiste dans ma peinture. Par nécessité profonde, par besoin. Je peux vivre par ailleurs des explosions d'intensité, les exprimer; lutter, tout bousculer, pour réorganiser ensuite... Le travail de l'artiste, comme je l'exprimais alors, s'opère dans un «équilibre en perpétuel renouvellement». Parlant de "l'art sans artifice» de Borduas, j'en soulignais «l'ordre, la proportion, l'équilibre, la nuance $" . .$.

J.-P.D. Dans l'un de ces articles, vous affirmiez que la contestation de l'artiste était aussi d'ordre politique.

F. L. Disons: d'ordre social. “Changer la vie", cela n'est pas seulement de l'ordre de la recherche artistique; le travail de l'artiste a des incidences - plus que des incidences - sur toute la vie. Nos positions politiques? Nous étions d'obédience marxiste, tout en n'étant pas d'accord avec un programme politique défini. C'était plutôt pour nous une attitude sociale: le cadre politique restreint, étriqué, de l'époque était le fait d'un monde de politiciens. Nous n'étions pas intéressés par la politique au sens strict. Nous étions en fait universalistes. Idéalistes, bien sûr. On aurait tendance aujourd'hui à identifier les Automatistes aux séparatistes québécois. II n'est pas dit que nous aurions opté pour la séparation, si la question s'était posée alors. Le politique est devenu une préoccupation majeure aujourd'hui. Nos idéaux sociaux de ce moment-là, globalement parlant, sont peut-être maintenant un peu incompréhensibles.

\section{J.-P. D. Vous parliez tout de même $d$ '«art national »...}

F. L. Oui, c'était à l'occasion d'une exposition itinérante des chefsd'cuvre de l'École hollandaise. Là encore, il faut se replacer dans le contexte. On nous parlait toujours d'un idéal hollandais (paysages, portraits). Et tout à coup arrivait cette grande exposition nous révélant les vrais maîtres, dans leur évolution. Nous avions soudain la révélation qu'il y avait eu une école hollandaise (c'est-à-dire un esprit de l'art hollandais) parce que la Hollande avait été un pays libre, puissant et 
riche de forces vitales, grâce à quoi s'était opéré le surgissement de nombreux artistes. Mais c'était là le fait d'une évolution d'ensemble, dans laquelle chaque artiste pouvait découvrir et vivre un aspect particulier de son pays, de son histoire ancienne et contemporaine. C'est le fait social d'un peuple qui, globalement, permet ou non l'éclosion et l'épanouissement d'une production artistique. Par exemple, une peinture nationale, pour nous, cela ne pouvait évidemment pas signifier des scènes de genre, des paysages enneigés ou des calèches... Encore une fois, le travail de l'artiste, son œuvre, ne sont pas séparables de l'évolution générale de son peuple. En Hollande, il y avait toute une richesse souterraine qui portait tout ce qui a donné la peinture hollandaise.

Quant à pouvoir ou non parler d'un art national au Québec aujourd'hui, je pense que c'est une fausse question; si l'on s'en tient à définir un pays en termes d'intégrité ou de spécificité, oui... peut-être. Mais aujourd'hui, l'art est universel. On trouve les mêmes formes au Japon, en Amérique. Au moment de l'automatisme, la situation était très différente: c'était la guerre, tout était fermé, cloisonné. Cela a favorisé un éveil national, la recherche d'une authenticité propre, et l'éclosion d'une peinture qui a été forcément «canadienne», avec des caractères spécifiques. Si l'on montait maintenant une exposition montrant toute la peinture canadienne, ou américaine, les œuvres se ressembleraient toutes, parce qu'on travaille dans un contexte de données universelles. Nous vivons à une époque d'échanges, de brassage total. La spécificité est autre chose désormais qu'un caractère «national » au sens étroit. Dans cette optique, le monde américain est l'un des phénomènes les plus importants d'après-guerre en peinture: c'est l'aventure, l'espace, les recherches dans tous les sens; il n'y a pas ici de tradition: tout le dynamisme à l'état brut. Dans ce sens, oui, il s'agirait d'un caractère «national» de la peinture américaine.

J.-P. D. Et au Québec, plus précisément, y a-t-il à l'heure actuelle des noyaux de jeunes peintres? Quelle impression gardez-vous, par exemple, de votre enseignement à Québec et à Montréal, il y a quelques années?

F. $L$ Je note certaines différences - toutes relatives il est vrai - entre les groupes d'étudiants qui ont suivi mon enseignement à Québec et à Montréal. Ces derniers sont plus cosmopolites, davantage américanisés. Ils ont vu plus de choses; ils en sont peut-être un peu blasés. A Québec, on trouve plus de naïveté, mais le potentiel me semble plus grand et les contacts humains sont plus profonds, même si l'on est plus refermé, plus «local» qu'à Montréal où il y a une plus grande ouverture. Mais la production des deux groupes, de toute façon, se ressent du grand brassage dont je parlais il y a un instant: il est impensable d'en rester au strict plan régional. La confrontation internationale est obligée. En fait, tout art, où qu'il soit, est "universel» en ce qu'il touche à l'homme; il est «international " par rapport aux grands courants qu'on retrouve partout dans le monde. 
J.-P. D. Claude Gauvreau dira plus tard de vous que vous aviez été un “grand lecteur et admirateur de Breton». Vous avez été en correspondance avec Breton à l'automne 1943; puis il vous a reçu à New York le $1^{\text {er }}$ avril 1945. Cette rencontre a été pour vous une profonde déception: pourquoi?

F. L. En fait, il n'y a eu qu'une lettre de part et d'autre. Et une seule rencontre. Breton était plus intéressé à "récolter" des disciples qu'à savoir ce que nous faisions à Montréal. Or de notre côté, nous avions déjà une certaine distance vis-à-vis du surréalisme: nous ne nous considérions pas du tout comme des disciples au sens strict. Je suis sorti de ma rencontre avec Breton très déçu, c'est vrai. Peut-être avais-je eu trop de naïveté? Je m'attendais à voir un personnage idéal; or, m'apparut un Breton très «réel», hautain, intellectuellement très fort certes, mais pour qui la magie surréaliste devait tenir surtout dans l'écriture. L'œuvre de Paul Klee nous paraissait, à nous, d'une importance capitale: Klee n'intéressait absolument pas Breton. Pour les Automatistes par contre, il n'était pas question d'introduire le littéraire, l'anecdote, dans le pictural. C'est vrai que nous avions été séduits, envoûtés par le surréalisme: toute la magie du jeu nous fascinait. Benjamin Péret, Lautréamont: une ouverture sur le merveilleux, sur le mystère. Mais si Breton était venu aux expositions de la rue Amherst ou de la rue Sherbrooke, il n'aurait vu là rien de surréaliste. Pour lui, la peinture devait être anecdotique, donner lieu au décodage possible d'une expression magique. Pour nous, il n'était pas question de cela: la peinture n'était pas porteuse de message, elle était langage direct, en soi. Cela dit, je garde toute mon admiration pour Breton: la déception que j'ai éprouvée au sortir de notre rencontre, j'en suis seul responsable. Au fond, la seule expression véritablement surréaliste de l'époque, à Montréal, ce fut le recueil de Thérèse Renaud: les Sables du rêve.

J.-P. D. Précisément, ces poèmes, illustrés de dessins par Mousseau, furent publiés aux Cahiers de la file indienne par Gilles Hénault et Éloi de Grandmont, en 1946. Le tirage était de trois cents exemplaires (les Sables du rêve seront réédités, trente ans plus tard, dans les Herbes rouges $n^{\circ} 29$; P. Haeck soulignera alors, dans le Devoir, l'assimilation des valeurs surréalistes, rêve, sexualité, et rappellera que ces poèmes passèrent totalement inaperçus à leur parution). Comment ce recueil a-t-il été composé? (C'est Thérèse Leduc qui répond.)

T. L. C'est assez étrange, l'histoire de ces poèmes: le livre a été l'objet d'une sorte d'occultation, d'abord à sa parution, et plus tard ensuite. Vers 1957-1958, Jean-Guy Pilon m'avait demandé quelques exemplaires des Sables pour un quelconque salon du livre à Montréal, et je les lui avais fournis: je n'en ai plus jamais entendu reparler. A l'époque, ma sœur Louise travaillait chez Pierre Matisse à New York, où elle était en contact avec les Surréalistes réfugiés aux États-Unis. Dans ses lettres, ma sœur me faisait le récit de ses rencontres. Cela dit, il faut préciser que j'écrivais dès les années 1942-1943, comme on écrit à quinze ans. 
Je me souviens que j'avais un "cahier à anneaux" dans lequel je notais des poèmes, des textes en prose, illustrés de dessins. Ce cahier est perdu aujourd'hui. Puis, Louise apporta à Montréal un exemplaire de la Sauterelle arthritique de Gisèle Prassinos, et elle me révéla la fameuse image de la terre "bleue comme une orange": ce fut littéralement pour moi comme un coup de foudre, une sorte de déclenchement, de catalyseur pour une écriture en train de naître. A partir de l'image d'Éluard, je réalisais qu'il était possible de créer une image poétique en exprimant une contre-vérité. Et je me mis à écrire de courts poèmes aux images incohérentes, mais qui exprimaient une réalité pour moi douloureuse: ce sont des poèmes tristes, déchirants même. A Paris en 1946, je fréquentais la Librairie du gai savoir où j'achetais les textes des Surréalistes et l'œuvre de Sade sous le comptoir. Je lus au libraire mes Sables du rêve, qu'il trouva étrangement "très drôles", et il voulut les passer au Seuil pour en faire une édition française. Je refusai. Encore une fois, c'était vraiment pour moi les poèmes d'une réalité tragique, traduisant un drame intérieur: je me sentais incomprise, inquiète, dans ın milieu familial bourgeois et éduquée chez les bonnes sceurs. II est vrai, par contre, que l'univers du jeu surréaliste représentait pour moi une immense libération, comme pour nos amis d'alors, et nous cherchions toujours à nous étonner les uns les autres. J'avais inventé, par exemple, “le cul grimaçant », entre autres facéties...

Pour revenir aux Sables du rêve, la composition du recueil lui-même s'est faite de manière assez fortuite: j'avais une pile de feuillets dactylographiés d'où nous tirions des poèmes au hasard, qui allaient constituer le recueil. J'en avais choisi quelques-uns que je voulais absolument voir publier, mais pour l'ensemble, c'est le hasard qui a décidé. Les autres sont perdus, comme mon premier cahier. Nous n'étions pas attachés aux choses, à laisser des traces: je lisais mes poèmes à nos amis, je chantais aussi ; c'est là que la poésie avait lieu. Le reste ne nous intéressait pas. Et puis, j'ai cessé d'écrire: ce mode de création poétique devenait facile. Je m'en suis désintéressée. Je ne suis revenue à l'écriture qu'en $1964^{2}$.

J.-P. D. Fernand Leduc, si l'on revient en 1945, après vos exercices d' "automatisme mécanique" qui entraînent alors Mousseau, Riopelle, Barbeau, vous en venez très rapidement dans votre peinture à rechercher "l'harmonie des couleurs" et "l'équilibre des volumes", comme le signale $B$. Teyssèdre. Donc: au moment où le mouvement automatiste est vraiment en marche, on assiste chez vous à une sorte de reprise par rapport au dynamisme, à l'informel et au gestuel purs... Quelle est alors votre voie, vers quoi tendaient désormais vos préoccupations?

F. L. II faut dire un mot de notre manifeste qui paraîtra à Montréal en août 1948. Avec le Refus global nous avons voulu faire le point sur notre expérience des quelques années qui précédaient, sur notre action de groupe. Là encore, c'est Borduas qui a été l'âme de ce manifeste; l'élaboration fut une entreprise commune, alors que Borduas assura la 
rédaction des textes eux-mêmes. Et puis, précision importante: avec la parution du Refus, l'automatisme était révolu pour moi. Peu après sa sortie, d'ailleurs, j'écrivis de Paris à Borduas en faisant une lecture critique de notre texte. Je venais de découvrir la pensée d'Abellio: pensée spiritualiste qui devait faire hausser les épaules à Borduas... Mais je voyais maintenant la pseudo-philosophie contenue dans le Refus glo$b a l$, et j'éprouvais le besoin de définir précisément les termes que nous avions utilisés. J'avais été séduit par la cosmogonie d'Abellio, et cela coincidait pour moi avec le besoin d'approfondir une nouvelle conformation du monde, un ordre global dont l'homme serait partie intégrante. J'étais à la recherche d'un point de vue supérieur. C'est dans cet état d'esprit que je fis la critique du Refus, à quoi Borduas réagit très froidement.

J.-P. D. Je retiens de cette lettre inédite quelques passages qui me paraissent capitaux: "Automatisme, sur-rationel, désir, passion, sensible, magie: autant de termes, il me semble, à reviser. [...] Les dualismes doivent disparaître. Déjà, "révolution " et «anarchie» n'ont plus de sens. Pas plus, d'ailleurs, que religion et morale.»

Un peu plus loin dans son texte, Fernand Leduc revient à la charge: “[...] De même de l'anarchie, de la révolution: il s'agit plutôt maintenant de hiérarchie, d'évolution (dans l'harmonie). " Cela constituait évidemment une sérieuse remise en question des affirmations passionnées du manifeste... Voire, une réfutation.

F. L. Pour moi, il ne pouvait plus être question d' «automatisme surrationnel». J'écrivais encore dans cette lettre: «L'automatisme, agent d'exécution dans notre activité picturale, est d'ordre rationnel. II ne peut être qu'une manière de tenir le pinceau, la plume, etc. Alors que le sur-rationnel est la qualité spirituelle organisatrice de l'activité même. » Donc, pour moi, le sur-rationnel devenait le point de départ, et l'automatisme le procédé d'exécution. Je disais à Borduas: “S'il faut une appellation, sur-rationnel se suffit. II nous conduit au domaine de l'esprit, de la connaissance, de la révélation." Cela n'allait pas trouver l'assentiment de Borduas, bien entendu.

Pour ce qui est de ma peinture, tandis que d'autres allaient dès lors vers l'éclatement de la forme, j'en suis venu graduellement au rassemblement des taches au profit de la forme, dans un processus d'organisation plus consciente. La spontanéité avait toujours son rôle à jouer, mais dans le sens de la spontanéité la plus immédiate de la réponse au désir du moment. J'allais peu à peu vers un monde d'organisation de la forme.

J.-P. D. Après un premier séjour à Paris (1947-1953), vous revenez à Montréal pour une assez longue période, de 1953 à 1959. Que se passet-il alors?

F. L. Entre 1948 et 1953, il y avait eu deux ou trois scandales dont l'âme dirigeante avait été Claude Gauvreau, approuvé par Borduas; par exem- 
ple, l'irruption des refusés au vernissage du Salon du musée de la rue Sherbrooke, avec déploiement de pancartes, en mars 1950. Le public et la critique avaient peu à peu tourné le dos à l'automatisme. II n'y avait plus eu d'expositions du groupe comme tel. En mai 1953, Roussil, Marcelle Ferron et moi organisions Place des artistes, au 82 ouest, rue Sainte-Catherine: une grande exposition réunissant les cuvres de plusieurs dizaines d'artistes de toutes tendances. Puis il y eut le collectif du Square Saint-Louis. Et je fondai I'Association des artistes non figuratifs de Montréal: il s'agissait de réunir, quelles que soient les tendances, des artistes de qualité, pour prouver au monde officiel de la peinture (Musée des beaux-arts, Ville de Montréal) que nous n'étions pas des sectaires, et que l'essentiel à nos yeux était bien les valeurs picturales. Progressivement, un groupe plus actif a pris l'initiative au sein de l'association: les Plasticiens. Claude Gauvreau, pour sa part, était resté envers et contre tout rivé à l'idée automatiste, avec quelques amis, surtout des littéraires. Les autres membres du groupe initial s'étaient engagés chacun dans sa propre direction, par exigence d'évolution et d'affirmation personnelles. Les voyages, les éloignements avaient également contribué à disperser le groupe automatiste.

J.-P.D. Comment avait évolué votre peinture, au cours de ces années? $B$. Teyssèdre définit votre production vers 1950 en parlant de "paysage non figuratif », de «style plus serein »...

F. L. "Paysage...»: oui, peut-être. Vers 1946, il y avait encore de l'espace dans mes tableaux. Puis j'en suis venu à grouper de plus en plus les éléments, à réduire l'espace, jusqu'à ne plus laisser subsister qu'une mince bordure entre les «pavés» de plus en plus importants: j'en arrivais à supprimer presque toute idée d'espace. Vers 1955 les masses occuperont toute la surface du tableau: c'est l'abstraction construite en plans géométriques.

J.-P. D. On peut se référer ici à l'excellente chronologie établie par Guy Viau dans le catalogue de votre exposition itinérante préparée par les Services extérieurs de la Galerie nationale du Canada, en 1970-1971, et dont l'organisateur était précisément Guy Viau, alors directeur du Centre culturel canadien à Paris.

II me semble qu'on trouve dans votre cheminement entre les années 1960 et 1970 comme un mouvement de simplification de la forme, jusqu'à l'extrême dépouillement des chromatismes binaires, des compositions, «passage» et «érosion»...

F. L. Oui. Ces jeux d'équilibre, positif et négatif, ou de plein et de vide, m'ont passionné. L'espace et la forme étaient, encore là, réduits à leur expression la plus ténue, la plus simple. J'avais toujours cette préoccupation de rapprocher les plans jusqu'à ce qu'ils se confondent presque totalement. Puis, une fois l'espace complètement éliminé, la forme le sera aussi. II ne restera que la restitution de la lumière, sans le support d'un espace, de formes, de volumes. Ce sera la microchromie. Une sorte 
de gageure, au fond: il persiste toujours en fait une forme sous-jacente qui porte la qualité de lumière qui est le tableau même. II ne s'agit pas de monochromie, comme on serait tenté de le croire à première vue.

J.-P. D. J'avais trouvé, citée par B. Teyssèdre, une phrase extraite d'un de vos articles du Quartier latin dont il a été question plus tôt dans notre entretien. Cette phrase me paraissait définir exactement, à trente ans de distance, vos grandes microchromies: "Seuls sont peintres ceux qui construisent, qui ordonnent par le dedans."

F. L. Oui, c'est assez amusant: les microchromies sont précisément construites, ordonnées «par le dedans", pour transposer la lumière variable dans ses aspects colorés... C'est la saisie d'une durée pure, sans forme ni structure apparente.

J.-P. D. Mais voilà que je découvre, sur le manuscrit original de l'article en question, une formulation de cette phrase quelque peu différente. Vous aviez écrit: "En peinture, il y a les créateurs: ceux qui construisent, qui ordonnent par le dedans, dans le sens de la vie. Eux seuls sont peintres." Et vous dénonciez alors les "amateurs de styles, de systèmes", les "assembleurs de matières précieuses, de formes étonnantes», les «ingénieurs-peintres" qui ne sont «ni ingénieurs ni peintres". Quoi qu'il en soit, où vous conduit maintenant cette recherche dont une étape importante a donné les microchromies?

F. L. On peut évidemment poser la question: et après? à chaque étape. J'ai abandonné les formes nettes et précises; je suis passé des couleurs pures aux tons rompus; de la rigidité à la ligne courbe hors de toute préoccupation de centrement du tableau. II y a constámment des problèmes nouveaux qui se posent. A l'heure actuelle, mes tableaux sont de plus en plus difficiles à faire et à percevoir, à cause des problèmes très ténus, très subtils qu'ils veulent résoudre. Je m'éloigne présentement du tableau unique qui se lirait comme une microchromie pour travailler sur des surfaces réunies en modules par six qui vibrent les uns par rapport aux autres en combinaisons multiples. Je n'ai pas fixé de données préalables: ces ordonnances se sont imposées d'ellesmêmes (lois de la couleur et de la lumière, interaction du chaud et du froid). Chaque panneau est un module interne, et les six se dynamisent l'un l'autre en valeurs complémentaires ou inversées soit à l'horizontale, à la verticale, en triangle ou en diagonale selon leur dialectique combinatoire propre. J'en suis ainsi arrivé à restituer toutes les lois de complémentarité des couleurs. Les tableaux permettent dix lectures de base, et d'autres encore: toutes les lois de la physique de la couleur sont réalisées.

A proprement parler, il n'y a pas «d'après": ça continue. "Après", c'est encore la vie, et non un mur, ou une rupture quelconque. Une curiosité en éveil est toujours à la recherche d'autre chose: il y a toujours une énigme à percer. Vouloir arriver à peindre la lumière, estce une folie? Oui, peut-être... Et puis, on n'a pas encore réussi à pein- 
dre l'énergie, par exemple... Cette recherche est très difficile, très lente; elle exige de plus en plus de patience, de réflexion. Si je peins quatre tableaux dans l'année au lieu de quinze, cela n'a pour moi aucune importance: je suis mon propre mouvement intérieur. Ça continue. Je rêve présentement de capter les qualités très particulières de la lumière grise de cette région, les brouillards de Champseru et de la plaine de Beauce. "Après", c'est une mauvaise question: ça continue.

1. Il faut se référer au numéro spécial de la Barre du jour sur "les Automatistes" (nos 17-18-19-20, janvier-août 1969). A défaut d'une étude systématique et exhaustive sur le groupe et l'époque, ce numéro renferme un certain nombre de textes et d'articles de premiere importance.

2. Thérèse Leduc travaille a ce moment-là à une fiction-autobiographie où elle analysait son rapport à la musique, à travers le thème de la recherche d'un maître. Ce manuscrit a été détruit. Puis, en 1966-1967, elle produit un texte sur la difficulté d'écrire. Plus récemment, elle écrit le Récit d'une errance, hiștoire d'un cheminement, d'une évolution intérieure, et qui a paru dans les Écrits du Canada français ( $\left.n^{\circ} 34,1972\right)$. Plus récemment, elle a écrit Mémoipe déchirée, lors d'un retour à Montréal après dix ans d'absence. Elle éprouvait le besoin de fixer le décor de son enfance, de revenir sur elle-même, sur son éducation et le début de sa jeunesse. 